\title{
Mass spectrometry-based serum proteomic signature as a potential biomarker for survival in patients with non-small cell lung cancer receiving immunotherapy
}

\author{
Young Kwang Chae ${ }^{1 \#}$, Won Bin Kim ${ }^{1 \#}$, Andrew A. Davis ${ }^{1}$, Lee Chun Park ${ }^{1,2}$, Jonathan F. Anker ${ }^{1}$, \\ Nicholas I. Simon ${ }^{1}$, Kyunghoon Rhee ${ }^{1}$, Junho Song ${ }^{1}$, Anderson Cho ${ }^{1}$, Sangmin Chang ${ }^{1}$, Taeyeong Ko ${ }^{1}$, \\ Michael $\mathrm{Oh}^{1}$, Manali Bhave ${ }^{1}$, Pedro Viveiros ${ }^{1}$ \\ ${ }^{1}$ Department of Medicine, Northwestern University Feinberg School of Medicine, Chicago, IL, USA; ${ }^{2}$ Division of Hematology/Oncology, Internal \\ Medicine, Kosin University, Busan, Republic of Korea \\ Contributions: (I) Conception and design: YK Chae, WB Kim; (II) Administrative support: YK Chae, WB Kim; (III) Provision of study materials or \\ patients: YK Chae, P Viveiros; (IV) Collection and assembly of data: WB Kim, LC Park, P Viveiros; (V) Data analysis and interpretation: WB Kim, \\ YK Chae, P Viveiros; (VI) Manuscript writing: All authors; (VII) Final approval of manuscript: All authors. \\ "These authors contributed equally to this work. \\ Correspondence to: Young Kwang Chae, MD, MPH, MBA. Northwestern Medicine Developmental Therapeutics Institute, 645 N. Michigan Ave., \\ Suite 1006, Chicago, IL 60611, USA. Email: young.chae@northwestern.edu.
}

\begin{abstract}
Background: VeriStrat test is a serum assay which uses a mass spectrometry (MS)-based proteomic signature derived from machine learning. It is currently used as a prognostic marker for patients with nonsmall cell lung cancer (NSCLC) receiving chemotherapy. However, little is known about its role for NSCLC patients receiving immune checkpoint inhibitors (ICIs).

Methods: This is a retrospective study that includes 47 patients with advanced stage NSCLC without an activating EGFR mutation, who underwent the VeriStrat test from 2016 to 2018. Spectra from blood samples were evaluated to assign patients into the VeriStrat 'Good' (VS-G) or VeriStrat 'Poor' (VS-P) risk group. The clinical outcomes of 32 patients who received programmed cell death 1 (PD-1) inhibitors nivolumab or pembrolizumab were analyzed by VeriStrat status.

Results: The VS-G group demonstrated significantly higher progression-free survival (PFS) and overall survival (OS) compared to the VS-P group among overall NSCLC patients regardless of treatment (median PFS of 7.1 vs. 4.2 months, $\mathrm{P}=0.013$, and median OS, not reached vs. 17.2 months, $\mathrm{P}=0.012$ ). Among NSCLC patients treated with ICIs, VS-G classification was associated with significantly increased PFS in comparison to VS-P classification (median PFS of 6.2 vs. 3.0 months, $\mathrm{P}=0.012$ ), while the differences in OS trended towards significance (median OS, not reached vs. 16.5 months $\mathrm{P}=0.076$ ). Multivariate analysis showed that the VeriStrat status was significantly correlated with PFS and OS in NSCLC patients treated with ICIs ( $\mathrm{P}=0.017, \mathrm{P}=0.034$, respectively).
\end{abstract}

Conclusions: MS-based serum proteomic signature has potential as a biomarker for survival outcome in NSCLC patients receiving immunotherapy.

Keywords: Non-small cell lung cancer (NSCLC); VeriStrat test; serum proteomic test; programmed death-1 (PD-1); immunotherapy

Submitted Jan 10, 2020. Accepted for publication Mar 13, 2020.

doi: $10.21037 /$ tlcr-20-148

View this article at: http://dx.doi.org/10.21037/tlcr-20-148 


\section{Introduction}

Lung cancer is the leading cause of cancer related death worldwide (1). The majority of patients with non-small cell lung cancer (NSCLC) is diagnosed in advanced stage with the historical five-year survival rate being less than $5 \%$. Except for patients who have actionable targets mutations with clearly defined drugs, patients receive either immunotherapy or a combination of immunotherapy and chemotherapy as first-line treatment (2-4).

Evidence has emerged demonstrating better survival outcomes and durable responses for patients using immune checkpoint inhibitors (ICIs) (2,5-12). Pembrolizumab, a PD-1 inhibitor, in combination with carboplatin and pemetrexed is approved as first-line treatment for patients with metastatic or advanced non-squamous NSCLC, regardless of PD-L1 expression $(13,14)$. While ICIs have shown promising results, a benefit in survival was only seen in a small subset of patients $(15-25 \%$ of all NSCLC patients) (15). There is a strong clinical need for biomarkers that can predict which patients will respond to ICIs.

Programmed cell death-ligand 1 (PD-L1) expression status on tumor cells is a widely accepted biomarker that has been used to predict the efficacy of anti-PD-1 and antiPD-L1 antibodies (6-8). However, a significant survival benefit has been observed even in PD-L1 negative patients and, on the other hand, not all patients with high PDL1 expression status are responsive to PD-1/PD-L1 inhibitors (16). These results can be explained by intratumoral heterogeneity of PD-L1 expression, changes in PD-L1 expression depending on the timing of biopsy collection, and different IHC assay methods (4). For these reasons, PD-L1 expression status on tumor cells may not be sufficient for patient stratification. Furthermore, tumor mutational burden (TMB), quantification of the mutations that result in new peptides or protein sequences in tumor cells, has also been correlated with response to PD-1/PDL1 inhibitors. Greater PFS was demonstrated in NSCLC patients with high TMB when treated with a combination of nivolumab and ipilimumab compared to those treated with chemotherapy in the first-line setting (17). However, many challenges remain including a lack of standard method for calculating TMB and controversies on the definition of the cut-off for different tumor types $(9,16)$. To improve the selection of patients who will most likely respond to immunotherapy as a single agent or in combination regimens, the development of new biomarkers is essential.

The VeriStrat test, a serum-based proteomic assay, is a multivariate tool that provides information regarding expected response to treatment as well as overall prognosis. It uses matrix-assisted laser desorption ionization time-offlight (MALDI-TOF) mass spectrometry (MS) to analyze serum samples of the patients to define eight mass spectra features (i.e., peaks). Peaks correspond to ions formed from abundant species such as peptides and proteins. Algorithms along with optimizations using machine learning are then performed to assign each spectrum to a VeriStrat 'Good' (VS-G) or VeriStrat 'Poor' (VS-P) classification (18). Based on this classification, the test can be used to predict which patients will experience longer survival in response to specific treatments. In comparison to PD-L1 expression status and TMB, the VeriStrat test has a standardized analysis procedure and only involves a peripheral blood draw. Furthermore, different MS tests with different cutoffs can be developed and validated for different subpopulations of immunotherapy treated NSCLC patients to predict survival outcomes more accurately.

The VeriStrat test's utility in predicting patient response has been validated in NSCLC patients treated with chemotherapy and targeted therapy in various conditions (18-26). In one study, seventy-six non-squamous lung cancer patients treated with standard first-line chemotherapy demonstrated that the VS-G patients had longer progression-free survival (PFS) and overall survival (OS) than VS-P patients (6.5 vs. 1.6 months, $\mathrm{P}<0.0001$, and 10.8 vs. 3.4 months, $\mathrm{P}<0.0001$, respectively). The VeriStrat status was found to be a statistically significant predictor of OS (25). In the phase III Lux-Lung 8 study in which a serum protein test was used to predict differential clinical outcomes with afatinib versus erlotinib, the OS was significantly longer in VS-G vs. VS-P patients, both in the overall population (HR 0.41, 95\% CI: 0.35-0.49) and in afatinib-treated patients (HR 0.40, 95\% CI: 0.31-0.51) (20). Furthermore, the prospective randomized phase III PROSE study demonstrated that patients classified as VS-P had better outcomes when receiving chemotherapy rather than erlotinib (23).

In addition to predicting survival outcomes, accurate prognostic measurements provided by the VeriStrat test help avoid ineffective, costly overtreatment and improve patient quality of life by precisely determining the appropriate time for optimal support and hospice care (27-30). A study that utilized a cost-effectiveness model concluded that the VeriStrat test reduced the use of active treatment by $9.1 \%$, changed the choice of active treatment in $20 \%$ of patients, improved OS by 0.7 months and 0.5 quality-adjusted 
months, and resulted in a net saving of $\$ 1,050$ per patient after factoring in the cost of the VeriStrat test (30). Patients who changed from erlotinib to chemotherapy on secondline treatment after using the proteomic test achieved an increase in quality-adjusted life years (QALYs) and saved costs (29). Among VS-P patients, the benefit was more substantial (27).

However, the VeriStrat classification has not been well studied in patients treated with immunotherapy. We hypothesize that the VeriStrat status has the potential to be a novel biomarker that can help determine which lung cancer patients will derive benefit from immunotherapy. The objective of this retrospective analysis was to evaluate whether there were differences in survival outcomes based on VeriStrat classification in patients treated with ICIs.

\section{Methods}

\section{Study design and end points}

This study was approved by The Institutional Review Board (IRB) of Northwestern University Feinberg School of Medicine (No. STU00205299). For this retrospective study, the written informed consent from patients was waived per the IRB, and the study was performed following the Health Insurance Portability and Accountability Act and the Declaration of Helsinki (as revised in 2013).

Seventy patients with advanced stage (III/IV) NSCLC at the Robert H. Lurie Comprehensive Cancer Center of Northwestern University underwent the VeriStrat test from 2016 to 2018. The median follow-up period was 17 months. Patients with EGFR mutation (n=8), patients lost to followup $(n=9)$, patients who received combination treatment of chemotherapy and immunotherapy $(n=5)$, and a patient whose result was determined to be VeriStrat-Indeterminate (VS-I) were not included in the study, resulting in the final sample size of 47 patients. For this study, patients were grouped into three sets for analyses: overall NSCLC patients $(n=47)$, NSCLC patients treated with ICIs $(n=32)$ and NSCLC patients treated with chemotherapy $(n=35)$. There was an intersection between the ICIs and chemotherapy groups because some patients received both treatments at different lines. This explains why the sum of the two groups do not add up to $(n=47)$. In this study, ICIs consisted of either the PD-1 inhibitor, pembrolizumab or nivolumab.

The survival outcome was assessed by analyzing PFS and OS for all three sets of patients. PFS was calculated from the start of first-line systemic treatment, ICI, or chemotherapy to disease progression or death in the overall NSCLC patients, NSCLC patients treated with ICIs and NSCLC patients treated with chemotherapy, respectively. OS was calculated from the start of first-line systemic treatment to the date of death, or in the case of NSCLC patients initially treated with ICIs, the start of ICIs to the date of death. In NSCLC patients treated with chemotherapy as first-line therapy, OS was calculated from the start of the chemotherapy to the date of death. Patients were censored if they were event-free at the final clinical assessment. Additionally, PFS and OS of patients treated with ICIs as first-line treatment $(n=8)$ were compared to those of patients treated with chemotherapy as first-line treatment $(n=35)$ to assess the predictive property of ICIs in comparison to standard chemotherapy.

\section{VeriStrat analysis}

Serum samples were collected from 2016 to 2018 and were frozen at $-80{ }^{\circ} \mathrm{C}$ until their use for mass spectrum generation for the VeriStrat test. The VeriStrat test was conducted by Biodesix (Boulder, CO, USA) according to the standard protocol previously described $(18,19)$. MALDI-TOF spectrometer was used to attain spectra of samples, which were then assigned a VS-G or VS-P risk group classification if all the replicates from the same sample resulted in the same classification; VS-I classification was assigned in the event of inconsistent classification.

\section{Statistical analysis}

Baseline characteristics of patients are presented as counts and percentages for categorical variables, and as mean and range for continuous variables. Comparison of continuous variables between the VeriStrat groups was performed using unpaired Student's $t$-test. Comparison of categorical variables was calculated using Fisher's exact test. To assess whether the VeriStrat classification was associated with clinical outcomes, the hazard ratios of PFS and OS were compared with forest plot, Microsoft Office Excel (Microsoft, Redmond, WA). Cox regression model was used to verify the VeriStrat test's prognostic value on PFS and OS while adjusting for other baseline factors, including gender, age, smoking status, ECOG performance status, histology, and prior treatment. These analyses were performed using SPSS Version 23 (SPSS Inc., Armonk, NY, USA).

For survival analyses, Kaplan-Meier curves were 
generated using the log-rank test. Time-to-event outcomes were analyzed using the data from patients who received at least one dose of first-line systemic therapy and were classified as VS-G or VS-P. These analyses were performed using GraphPad Prism 7 (La Jolla, CA, USA).

For this study, the same statistical analyses were performed in the main three subsets of patients: overall NSCLC patients ( $\mathrm{n}=47)$, NSCLC patients treated with ICIs $(n=32)$ and NSCLC patients treated with chemotherapy $(\mathrm{n}=35)$. Additionally, a similar analysis was tried for NSCLC patients who underwent the VeriStrat test before ICIs $(\mathrm{n}=12)$, and NSCLC patients who underwent the VeriStrat test before chemotherapy $(\mathrm{n}=10)$.

\section{Results}

\section{Patient baseline characteristics}

Forty-seven patients were included in the study. Thirtytwo patients were classified as VS-G, and fifteen patients were classified as VS-P. Table 1 shows the distribution of clinical characteristics by the VeriStrat test classification. The patient population had a mean age of 65.7 years (range, $30-91$ years), 25 patients $(53.2 \%)$ were female, and 22 patients $(46.8 \%)$ were male. By the time of the analysis, 17 patients $(36.2 \%)$ had died (nine and eight in VS-G and VS-P group, respectively). The majority of patients (85.1\%) were former or current smokers and most (76.6\%) had stage IV NSCLC. There were significantly more squamous cell carcinoma cases among VS-P than among VS-G patients ( $60.0 \%$ vs. $25.0 \%$, respectively, $\mathrm{P}=0.027$, Table 1$)$. Thirtytwo patients $(68.1 \%)$ had undergone treatment with ICIs at the time of analyses, whereas 15 patients $(31.9 \%)$ had not. Of the overall 47 analyzed patients, 16 had undergone other treatment modalities such as radiation and surgery before chemotherapy or ICIs. Of the 32 NSCLC patients treated with ICIs, 12 were subject to the VeriStrat test before ICIs (Table S1). Of the 35 NSCLC patients treated with chemotherapy, 10 were subject to the VeriStrat test before chemotherapy.

The majority of patients received ICIs as second or thirdline treatment $(\mathrm{n}=24)$ after receiving first-line treatment such as chemotherapy $(\mathrm{n}=35)$ (Table 2). Furthermore, more NSCLC patients treated with ICIs were subject to the VeriStrat test after the start of treatment with ICIs (62.5\%). The percentage of VS-P patients was higher among patients who were subject to the VeriStrat test after the start of treatment with ICIs $(50.0 \%)$ than for those who were subject to it before the start of ICI treatment (33.3\%), but the difference was not significant $(\mathrm{P}=0.471$, Table S1).

\section{Survival in overall NSCLC patients by serum proteomic signature}

VS-G patients in the overall NSCLC population had a $65 \%$ lower risk of progression ( $\mathrm{HR}=0.35,95 \% \mathrm{CI}: 0.15-0.82$ ) and $78 \%$ lower risk of death (HR $=0.22,95 \%$ CI: $0.07-0.72$ ) compared to VS-P patients (Figure 1). The median PFS was 7.1 months for VS-G patients, significantly longer than the 4.2 months for VS-P patients $(\mathrm{P}=0.013)$ (Figure $2 A)$. VS-G did not reach median OS as survival exceeded $50 \%$ at the time of analysis, which was significantly longer than 17.2 months by VS-P patients $(\mathrm{P}=0.012)$ (Figure $2 B)$. Multivariate analysis showed that the VeriStrat status was significantly correlated with PFS, after adjusting for clinical factors such as age ( $\mathrm{HR}=0.38,95 \% \mathrm{CI}$ : $0.16-0.88, \mathrm{P}=0.024$ ) (Table S2). Also, the VeriStrat status was significantly correlated with OS (HR $=0.21,95 \% 0.05-0.89, \mathrm{P}=0.035$ ) (Table S3).

\section{Survival in NSCLC patients treated with ICIs by serum proteomic signature}

VS-G patients treated with ICIs had a $72 \%$ lower risk of progression compared to VS-P patients (HR $=0.28,95 \%$ CI: $0.11-0.76, \mathrm{P}=0.012$, Figure 1). The median PFS was 6.2 months for VS-G patients, significantly longer than the 3.0 months for VS-P patients (Figure 2C). The median OS of VS-G in NSCLC patients treated with ICIs was longer than VS-P with a trend toward significance $(\mathrm{P}=0.076)$ (Figure 2D). Multivariate analysis determined that the VeriStrat status was significantly correlated with PFS (HR $=0.26,95 \%$ CI: $0.08-0.79, \mathrm{P}=0.017)$ and $\mathrm{OS}(\mathrm{HR}=0.16$, 95\% CI: $0.03-0.87, \mathrm{P}=0.034)$ among patients treated with ICIs (Tables 3 and 4, respectively).

\section{Survival in NSCLC patients treated with chemotherapy by serum proteomic signature}

VS-G patients in the NSCLC patients treated with chemotherapy had a $61.1 \%$ lower risk of PFS (HR $=0.39,95 \%$ CI: $0.15-0.99)$ and an $81.0 \%$ lower risk of OS (HR $=0.19,95 \%$ CI: 0.05-0.76) compared to VS-P patients (Figure 1). In the NSCLC patients treated with chemotherapy, VS-G patients had significantly longer median PFS than VS-P patients (Figure 2E). A multivariate 
Table 1 Baseline characteristics of patients by the VeriStrat test classification

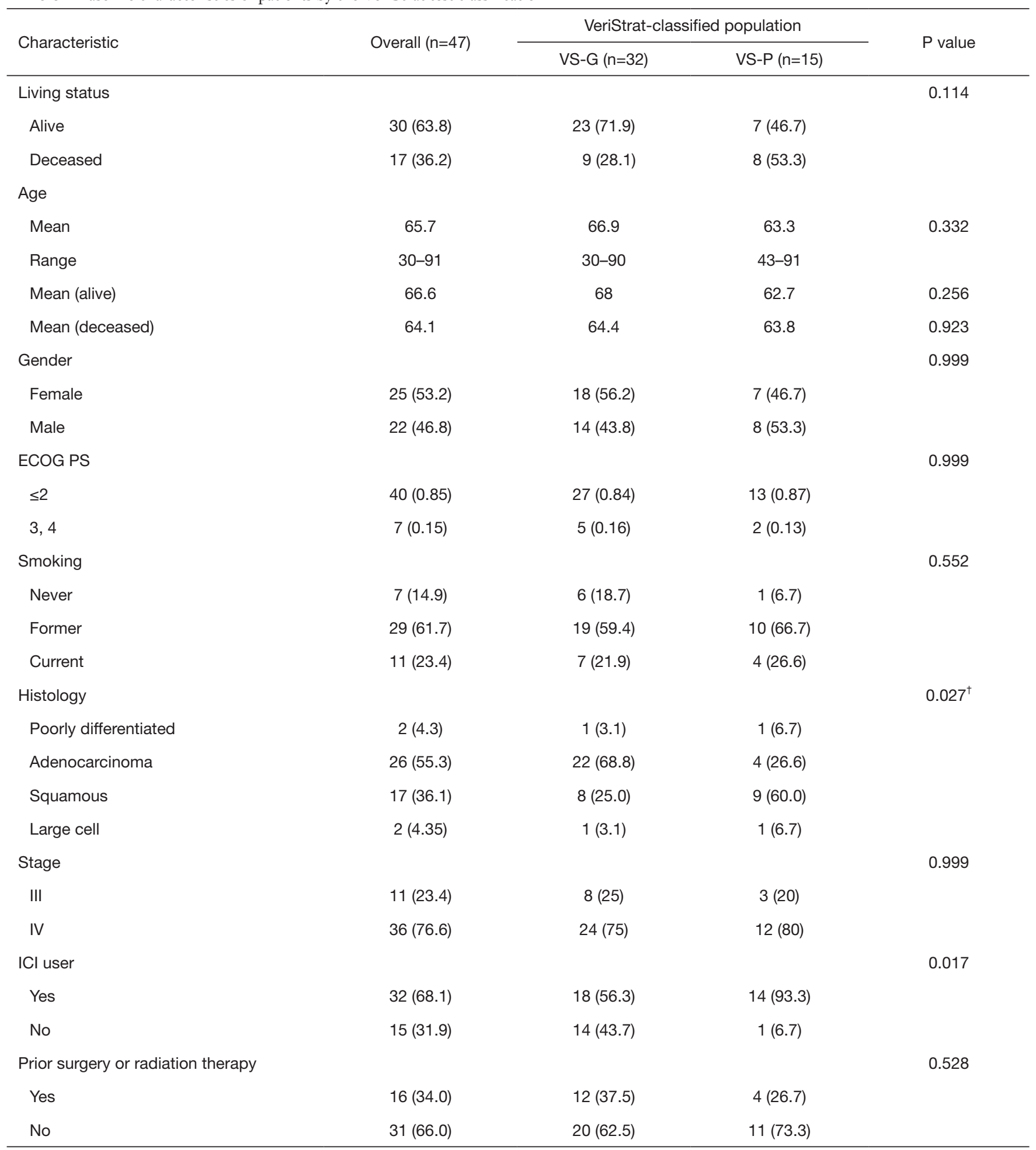

Data given as No. (\%) unless otherwise indicated. ${ }^{\dagger}$, squamous vs. non-squamous. ECOG PS, Eastern Cooperative Oncology Group Performance Status; VS-G, VeriStrat Good; VS-P, VeriStrat Poor; ICI, immune-checkpoint inhibitor. 
Table 2 Status of ICI treated patients by the VeriStrat test classification

\begin{tabular}{lccc}
\hline \multirow{2}{*}{ Line of treatment } & Overall $(\mathrm{n}=32)$ & \multicolumn{2}{c}{ VeriStrat-classified population } \\
\cline { 3 - 3 } & & VS-G $(\mathrm{n}=18)$ & VS-P $(\mathrm{n}=14)$ \\
\hline $\mathrm{ICl}$ & $8(25.0)$ & $4(22.2)$ & $4(28.6)$ \\
1st line & $17(53.1)$ & $8(44.5)$ & $9(64.3)$ \\
2nd line & $7(21.9)$ & $6(33.3)$ & $1(7.1)$ \\
3rd line & & & \\
\hline
\end{tabular}

Data given as No. (\%) unless otherwise indicated. ICI, immune-checkpoint inhibitor; VS-G, VeriStrat Good; VS-P, VeriStrat Poor.

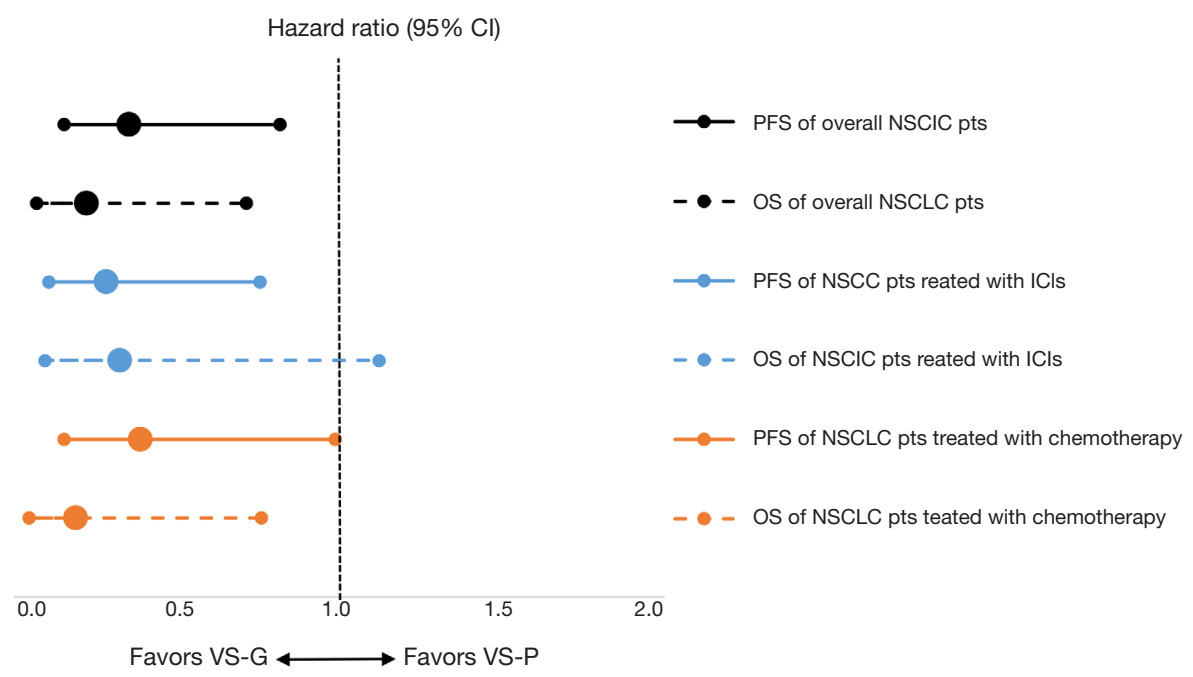

Figure 1 Forest plot by the VeriStrat test classification. Hazard ratio of 1 suggests no difference in survival outcomes between patients with VS-G and VS-P classification. Hazard ratio towards the left of the dotted line favors VS-G, whereas a hazard ratio greater than 1 favors VS-P. CI, confidence interval; PFS, progression-free survival; OS, overall survival; pts, patients; NSCLC, non-small cell lung cancer; ICIs, immune checkpoint inhibitors; VS-G, VeriStrat Good; VS-P, VeriStrat Poor.

analysis displayed a trend toward significance for VeriStrat status being correlated with PFS for these patients, while adjusting for other covariates ( $\mathrm{HR}=0.38,95 \%$ CI: $0.12-1.15, \mathrm{P}=0.087$ ) (Table S4). Furthermore, VS-G was associated with longer median OS when compared with VS-P in the overall NSCLC patients treated with chemotherapy (Figure $2 F$ ). A multivariate analysis displayed a trend for VeriStrat status being significantly correlated with OS (HR $=0.18,95 \%$ CI: 0.03-1.12, $\mathrm{P}=0.065)$ (Table S5).

\section{Survival in NSCLC patients who received the VeriStrat test before the treatment}

The median PFS and OS were not significantly different between VS-G and VS-P patients who were subject to the VeriStrat test before the start of ICIs (Figure $3 A, B$, respectively). In contrast, VS-G was associated with longer median PFS and OS when compared with VS-P in patients who were subject to the VeriStrat test before the start of chemotherapy (Figure $3 C, D$, respectively).

\section{Survival in NSCLC patients treated with ICIs versus chemotherapy as first-line treatments}

When projecting the survival curves for VS-G patients, PFS distribution was not significantly different between the ICIs and chemotherapy treated groups (Figure 4A). The median PFS of patients treated with ICIs was 7.4 months while that of patients treated with chemotherapy was 7.0 months 
A

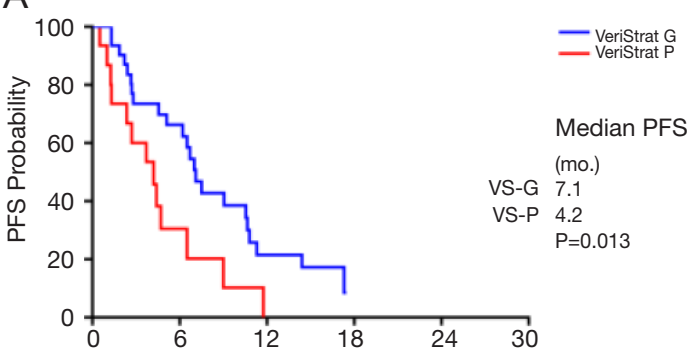

Number at risk Time of Progression-Free Survival (months)

$\begin{array}{lllllll}\text { VS-G } & 32 & 19 & 6 & 1 & 1 & 1\end{array}$
C

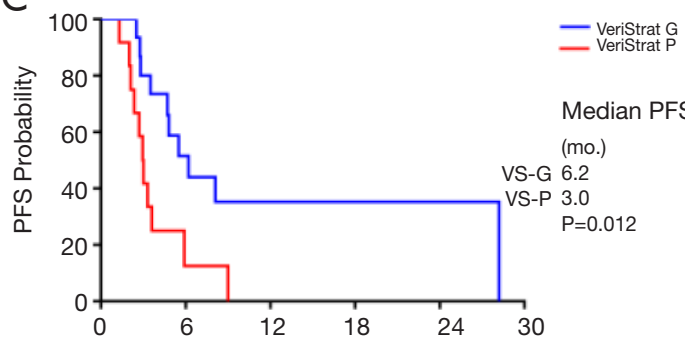

Number at risk

Time of Progression-Free Survival (months)
B

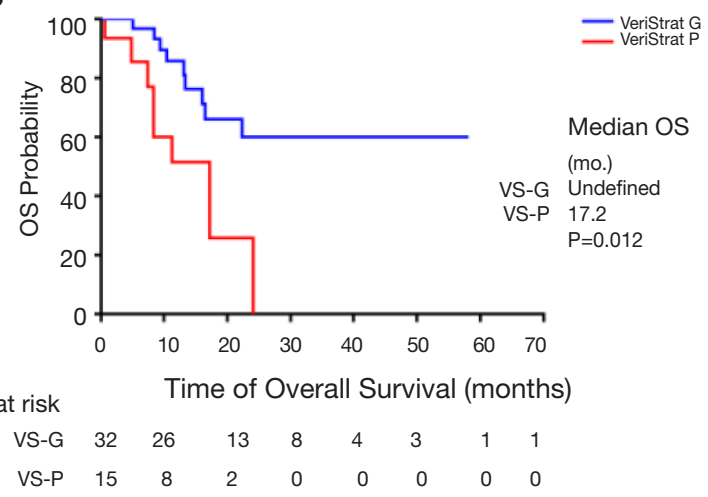

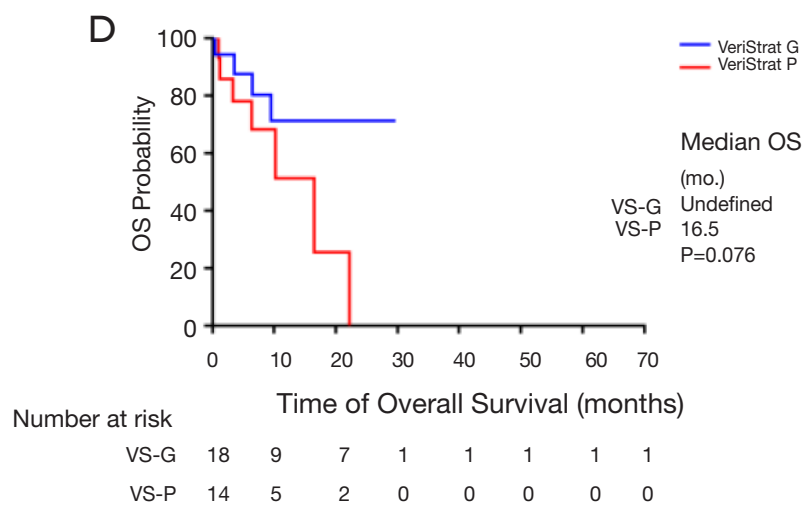

E 二 Zeristrat $G_{\text {Veristrat P }}$

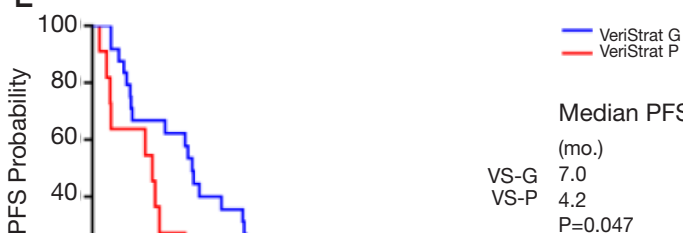

Number at risk Time of Progression-Free Survival (months)

$\begin{array}{lllllll}\text { VS-G } & 24 & 15 & 5 & 1 & 1 & 1 \\ \text { VS-P } & 11 & 3 & 1 & 0 & 0 & 0\end{array}$

$\mathrm{F}$

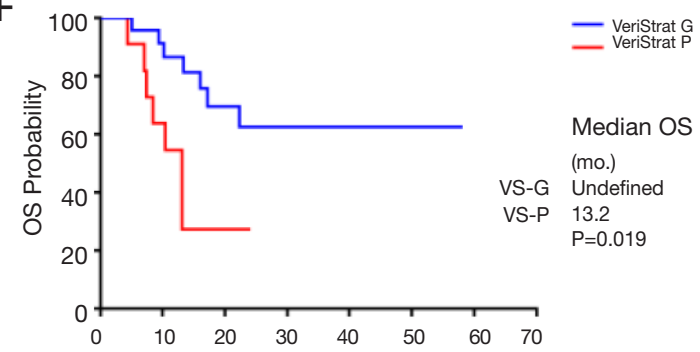

Number at risk

Time of Overall Survival (months)

$\begin{array}{lllllllll}\text { VS-G } & 24 & 21 & 12 & 7 & 4 & 3 & 1 & 1\end{array}$

$\begin{array}{lllllllll}\text { VS-P } & 11 & 8 & 2 & 1 & 1 & 1 & 1 & 1\end{array}$

Figure 2 Survival curves of NSCLC patients by the VeriStrat classification. (A) PFS of the overall NSCLC population; (B) OS of the overall NSCLC population; (C) PFS of NSCLC population treated with ICIs; (D) OS of NSCLC population treated with ICIs; (E) PFS of NSCLC population treated with chemotherapy; (F) OS of NSCLC population treated with chemotherapy. PFS, progression-free survival; OS, overall survival; NSCLC, non-small cell lung cancer; ICIs, immune checkpoint inhibitors; VS-G, VeriStrat Good; VS-P, VeriStrat Poor. 
Table 3 Cox proportional hazard models of progression-free survival by NSCLC patients treated with immune checkpoint inhibitors

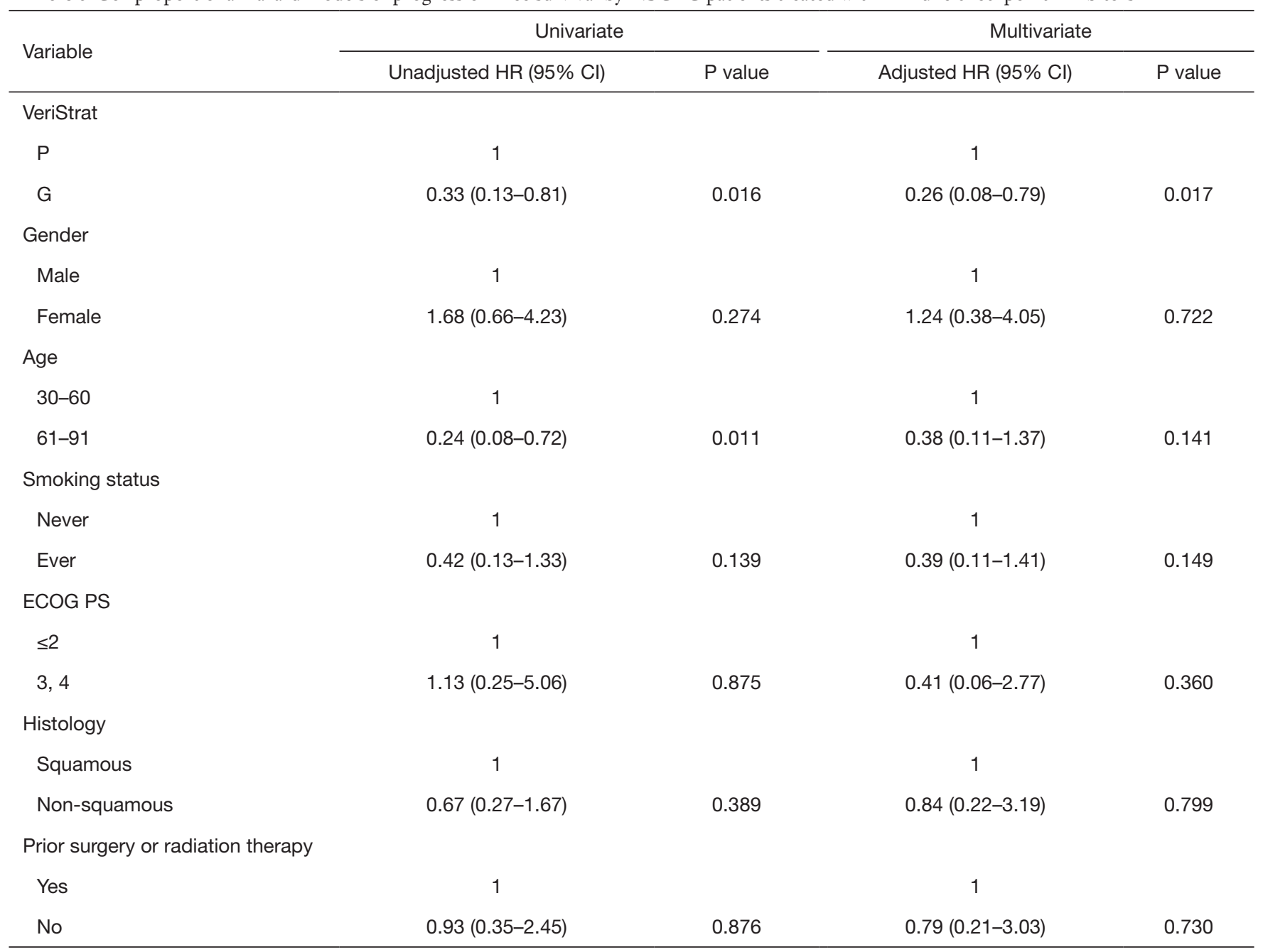

NSCLC, non-small cell lung cancer; Cl, confidence interval; HR, hazard ratio; VS-G, VeriStrat Good; VS-P, VeriStrat Poor; ECOG PS, Eastern Cooperative Oncology Group Performance Status.

(HR 0.62, 95\% CI: 0.13-3.06, $\mathrm{P}=0.556$ ). There was also no significant difference in PFS between treatment groups for the VS-P patients (HR 0.83, 95\% CI: 0.23-3.01, $\mathrm{P}=0.783$ ). Similarly, OS was not significantly different between the ICI and chemotherapy-treated VS-G patients (Figure 4B). The median OS of the patients treated with ICIs was 22.3 months while that of patients treated with chemotherapy was not reached (HR 1.71, 95\% CI: 0.14-21.19, $\mathrm{P}=0.675$ ). There was no significant difference in OS between the treatment groups in the VS-P patients (HR 0.61, 95\% CI: $0.13-2.89, \mathrm{P}=0.534)$.

\section{Discussion}

The VeriStrat test is a serum-based proteomic test that has been shown to have potential application in guiding decision making for patients undergoing chemotherapy and targeted therapy but has been studied to a limited extent in NSCLC patients undergoing immunotherapy. In this study, we assessed whether the VeriStrat status could be a prognostic and predictive biomarker test in NSCLC patients treated with ICIs. In a retrospective analysis of NSCLC patients, we found that patient classifications based on the VeriStrat test were prognostic for PFS in advanced NSCLC patients treated with ICIs. Our results show that the VeriStrat test, which was developed initially as a biomarker for NSCLC patients treated with EGFR TKIs, has the potential to be used as a biomarker for NSCLC patients treated with ICIs.

Numerous studies support the clinical utility of the VeriStrat test but the mechanistic connection between 
Table 4 Cox proportional hazard models of overall survival by NSCLC patients treated with immune checkpoint inhibitors

\begin{tabular}{|c|c|c|c|c|}
\hline Variable & \multicolumn{2}{|c|}{ Univariate } & \multicolumn{2}{|c|}{ Multivariate } \\
\hline \multicolumn{5}{|l|}{ Veristrat } \\
\hline $\mathrm{P}$ & 1 & & 1 & \\
\hline $\mathrm{G}$ & $0.34(0.10-1.18)$ & 0.089 & $0.16(0.03-0.87)$ & 0.034 \\
\hline \multicolumn{5}{|l|}{ Gender } \\
\hline Male & 1 & & 1 & \\
\hline Female & $1.42(0.41-4.92)$ & 0.578 & $1.33(0.24-7.34)$ & 0.743 \\
\hline \multicolumn{5}{|l|}{ Age } \\
\hline $30-60$ & 1 & & 1 & \\
\hline \multicolumn{5}{|l|}{ Smoking status } \\
\hline Never & 1 & & 1 & \\
\hline Ever & $1.34(0.17-10.74)$ & 0.784 & $0.93(0.10-8.44)$ & 0.947 \\
\hline \multicolumn{5}{|l|}{ ECOG PS } \\
\hline$\leq 2$ & 1 & & 1 & \\
\hline 3,4 & $0.99(0.12-7.86)$ & 0.988 & $0.42(0.03-5.64)$ & 0.514 \\
\hline \multicolumn{5}{|l|}{ Histology } \\
\hline Squamous & 1 & & 1 & \\
\hline Non-squamous & $1.79(0.53-6.03)$ & 0.350 & $3.36(0.74-15.36)$ & 0.118 \\
\hline
\end{tabular}

Cl, confidence interval; HR, hazard ratio; VS-G, VeriStrat Good; VS-P, VeriStrat Poor; ECOG PS, Eastern Cooperative Oncology Group Performance Status.

VeriStrat poor classification and poor prognosis in untreated and previously treated patients still remains an area for active research. Multiple isoforms of serum amyloid A1 (SAA1), acute phase proteins, were identified as the main proteins that generate 4 out of 8 mass signals composing the proteomic algorithm of VeriStrat (31). In fact, a study that collected samples from 57 patients receiving salvage chemotherapy and 70 non-EGFR mutated patients receiving erlotinib demonstrated that VeriStrat poor classification was significantly associated with proinflammatory/acute phase reactants including higher C-reactive protein, serum amyloid A, Cyfra 21-1, ferritin, osteopontin, and lower IGF-II (32). These proinflammatory reactants are known to be associated with carcinogenesis and poor prognosis in cancer patients (33). Similarly, we anticipate that $v s$. poor patients may receive less benefit from ICI therapy due to presence of more molecules and pathways associated with tumor progression and treatment resistance. More studies need to identify mechanisms at molecular level to validate VeriStrat test's prognostic and predictive power to improve outcome in NSCLC patients classified as VeriStrat poor.

According to the FDA-NIH Biomarker working group, a prognostic biomarker provides information about the likelihood of a clinical event, disease recurrence, or disease progression, regardless of therapy, while a predictive biomarker measures the effect of a medical therapy (34). The VeriStrat status has proven to be both prognostic and predictive in patients treated with chemotherapy and 

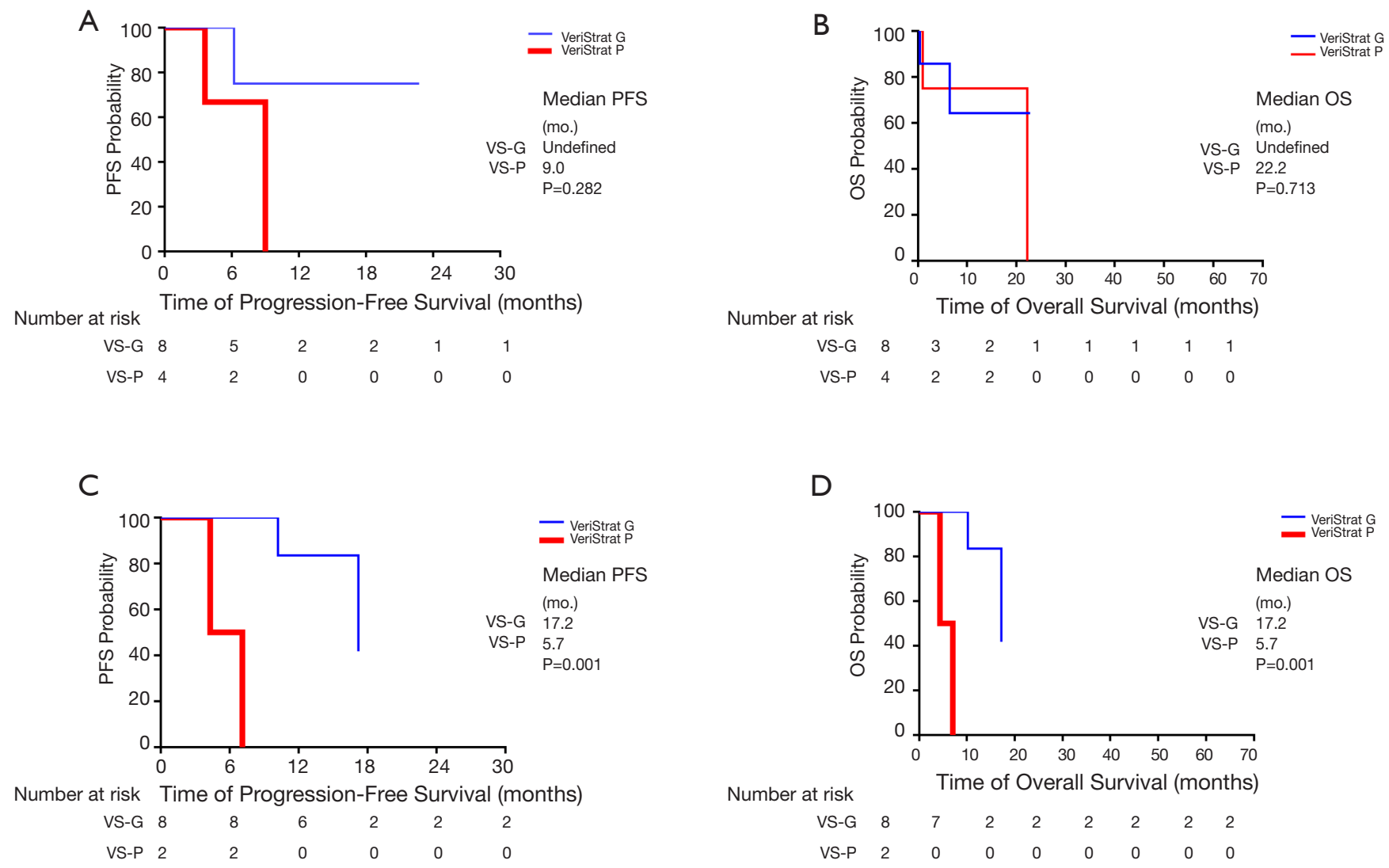

Figure 3 Survival curves of NSCLC patients who received the VeriStrat test before the treatment. (A) PFS of NSCLC population who received the VeriStrat test before the start of ICIs; (B) OS of NSCLC population who received the VeriStrat test before the start of ICIs; (C) PFS of NSCLC population who received the VeriStrat test before the start of chemotherapy; (D) OS of NSCLC population who received the VeriStrat test before the start of chemotherapy. PFS, progression-free survival; OS, overall survival; NSCLC, non-small cell lung cancer; ICI, immune checkpoint inhibitor; VS-G, VeriStrat Good; VS-P, VeriStrat Poor.

A

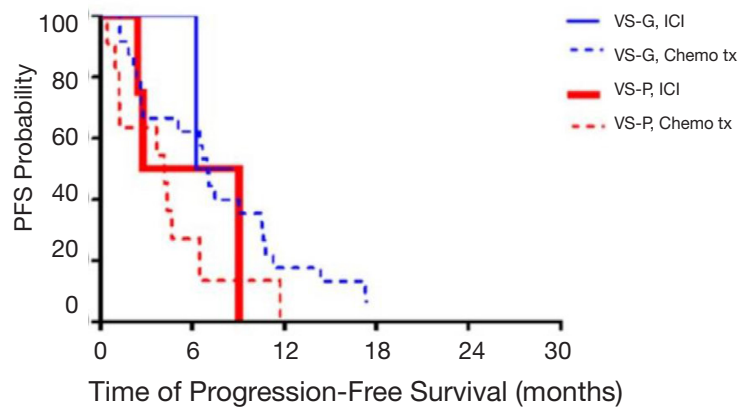

B

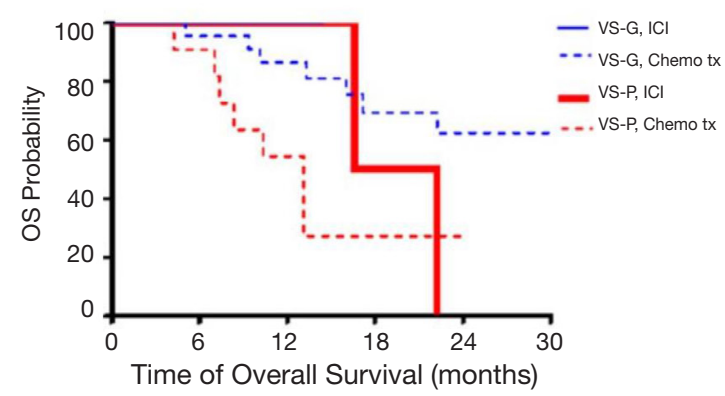

Figure 4 Survival curves of NSCLC patients treated with ICIs versus chemotherapy as first-line treatments. (A) Progression-free survival curves; (B) overall survival curves. NSCLC, non-small cell lung cancer; ICI, immune checkpoint inhibitor. 
targeted therapy (19,21-26). Studies including the NExUS trial verified that the VeriStrat test could identify patients who would go on to have better clinical outcome from firstline platinum doublet therapy. These studies demonstrated that patients with the VS-G classification were likely to derive benefit from a first-line platinum-based therapy, while those with a VS-P classification were less likely to respond to platinum-based therapy (24,25). Additionally, the BR.21 phase III trial, which examined erlotinib versus placebo in previously treated advanced NSCLC patients, demonstrated that VS-G patients had a significantly longer OS as compared with VS-P patients in erlotinib-treated NSCLC patients, independent of clinical covariates (19). In agreement with these previous studies, our study demonstrated that the VS-G classification strongly correlated with longer PFS and OS compared to the VS-P classification in the overall pool of NSCLC patients $(n=47)$, which includes patients treated with ICIs. The VeriStrat classification was an independent prognostic indicator for PFS and OS in the multivariate analysis.

The prognostic property of the serum-proteomic test for NSCLC patients treated with ICIs was studied for the first time in a study of NSCLC patients treated with second-line nivolumab (35). The study comprised of sixty previously treated advanced NSCLC patients and the VeriStrat test of pre-treatment patients demonstrated that the VeriStrat status was prognostic for OS. Similarly, our study was also able to identify that patients with advanced NSCLC treated with ICIs and a VS-G classification were likely to have a trend towards increased OS when compared with VS-P patients. Furthermore, our study demonstrated that the VeriStrat status is prognostic for PFS among NSCLC patients treated with ICIs. VeriStrat may still hold its prognostic features for ICIs-treated NSCLC patients.

A previous study on the VeriStrat test in the course of EGFR TKIs therapy suggested that about one third of baseline VS-G classifications will change to the VS-P classification during the course of treatment (36). We ran a separate analysis on survival outcomes for patients who received the VeriStrat test before the start of ICIs, but the small number of patients in each VeriStrat classification subgroup made the results exploratory. Our results could not demonstrate that the VeriStrat test, when obtained preimmunotherapy, is a significant prognostic indicator of PFS or OS for NSCLC patients treated with ICIs, though the inadequate sample size interferes with drawing conclusions. Another concern was that lack of sufficient follow-up time could harm the visualization of differences in long-term survival between the VeriStrat classification groups. The median follow-up period of the VeriStrat test was seventeen months, and over half of the VS-G patients were alive at the point of analysis. A longer follow-up period could reveal significant differences between VS-G and VS-P patients regarding OS, in place of the current results.

The predictive property of the VeriStrat test has been demonstrated in various studies, including the phase III PROSE study, and the BR.21 trial, the latter of which verified that erlotinib should be used in VS-G classified previously treated advanced NSCLC patients $(19,21,23)$. The median survival was 10.5 months on erlotinib $v$ s. 6.6 months for placebo in VS-G patients (HR 0.63, 95\% CI: $0.47-0.85, \mathrm{P}=0.002)$. The VeriStrat status was predictive for objective response to erlotinib $(\mathrm{P}=0.002)$. Furthermore, the prospective randomized phase III PROSE study, which was comprised of stage IIIB or IV NSCLC patients, demonstrated that patients classified as VS-P should select chemotherapy over erlotinib as second-line therapy. VS-P patients had worse survival on erlotinib than on chemotherapy (HR 1.72, 95\% CI: 1.08-2.74, $\mathrm{P}=0.022$ ), whereas VS-G patients had similar OS with either regimen. In our study, the VeriStrat status could not predict differences in survival outcomes in ICIs relative to chemotherapy, as the difference in PFS and OS for VS-G patients were not significant between those receiving ICIs and those receiving chemotherapy as first-line treatments. However, our result on the predictive power of the VeriStrat status was limited, as there were only eight patients who received ICIs as first-line treatment compared to thirty-five patients who received chemotherapy.

Inherent limitations of our study include the retrospective nature of this analysis and the relatively small number of patients $(n=47)$. We would have favored in limiting our analysis to patients who were subject to the VeriStrat test before the start of systemic therapy, as previous treatment lines may influence results. Unfortunately, those patients were the minority in our cohort.

In addition to NSCLC patients, a study on melanoma patients has provided a proof of concept that serum proteomic test like the VeriStrat test can potentially serve as a prognostic biomarker test for patients with other malignancies treated with ICIs $(37,38)$. A study on stage IV melanoma patients treated with nivolumab $(\mathrm{n}=119)$ reported protein signatures that predicted survival outcome using MS analysis and machine-learning. Similar to these efforts, optimized serum proteomic tests can be developed to predict the NSCLC patients who will likely respond to 
ICIs. First however, the VeriStrat test needs to be evaluated on whether it is a prognostic test of survival outcomes in NSCLC patients treated with ICIs.

Future trials are warranted that stratify patients according to serum proteomic signature in order to identify durable responders for ICI-treated NSCLC patients. From those results, new treatment strategies could be attempted for poor prognosis patients.

\section{Conclusions}

There has been limited research assessing the clinical utility of serum-based proteomic testing in NSCLC patients treated with immunotherapy. This retrospective study evaluated the prognostic and predictive properties of the VeriStrat test and demonstrated that the VS-G status is associated with a favorable PFS compared to VS-P classification in NSCLC patients treated with ICIs. The VeriStrat status could serve as a potential biomarker for patients undergoing treatment with ICIs and may therefore improve treatment decision-making for advanced NSCLC patients.

\section{Acknowledgments}

Funding: None.

\section{Footnote}

Conflicts of Interest: All authors have completed the ICMJE uniform disclosure form (available at http://dx.doi. org/10.21037/tlcr-20-148). YKC would like to disclose advisory and consulting for AstraZeneca, Genentech, Takeda Pharmaceuticals, Guardant Health and Foundation Medicine. YKC is participating in research projects with Abbvie, BMS, Lexent Bio, Freenome, and Biodesix. The other authors have no conflicts of interest to declare.

Ethical Statement: The authors are accountable for all aspects of the work in ensuring that questions related to the accuracy or integrity of any part of the work are appropriately investigated and resolved. This study was approved by The Institutional Review Board (IRB) of Northwestern University Feinberg School of Medicine (No. STU00205299). For this retrospective study, the written informed consent from patients was waived per the IRB, and the study was performed following the Health Insurance Portability and Accountability Act and the Declaration of
Helsinki (as revised in 2013).

Open Access Statement: This is an Open Access article distributed in accordance with the Creative Commons Attribution-NonCommercial-NoDerivs 4.0 International License (CC BY-NC-ND 4.0), which permits the noncommercial replication and distribution of the article with the strict proviso that no changes or edits are made and the original work is properly cited (including links to both the formal publication through the relevant DOI and the license). See: https://creativecommons.org/licenses/by-nc-nd/4.0/.

\section{References}

1. Ferlay J, Soerjomataram I, Dikshit R, et al. Cancer incidence and mortality worldwide: sources, methods, and major patterns in GLOBOCAN 2012. Int $\mathrm{J}$ Cancer 2015;136:E359-86.

2. West H. Nivolumab as first line monotherapy for advanced non-small cell lung cancer: could we replace first line chemotherapy with immunotherapy? Transl Lung Cancer Res 2014;3:400-2.

3. PDQ Adult Treatment Editorial Board. Non-Small Cell Lung Cancer Treatment (PDQ®)-Health Professional Version. Available online: https://www.ncbi.nlm.nih.gov/ books/NBK65865/. Accessed April 3, 2018.

4. Yoneda K, Imanishi N, Ichiki Y, et al. Immune Checkpoint Inhibitors (ICIs) in Non-Small Cell Lung Cancer (NSCLC). J UOEH 2018;40:173-89.

5. Fehrenbacher L, Spira A, Ballinger M, et al. Atezolizumab versus docetaxel for patients with previously treated non-small-cell lung cancer (POPLAR): a multicentre, open-label, phase 2 randomised controlled trial. Lancet 2016;387:1837-46.

6. Rittmeyer A, Barlesi F, Waterkamp D, et al. Atezolizumab versus docetaxel in patients with previously treated non-small-cell lung cancer (OAK): a phase 3, openlabel, multicentre randomised controlled trial. Lancet 2017;389:255-65.

7. Borghaei H, Paz-Ares L, Horn L, et al. Nivolumab versus Docetaxel in Advanced Nonsquamous Non-Small-Cell Lung Cancer. N Engl J Med 2015;373:1627-39.

8. Brahmer J, Reckamp KL, Baas P, et al. Nivolumab versus Docetaxel in Advanced Squamous-Cell Non-Small-Cell Lung Cancer. N Engl J Med 2015;373:123-35.

9. Carbone DP, Reck M, Paz-Ares L, et al. First-Line Nivolumab in Stage IV or Recurrent Non-Small-Cell Lung Cancer. N Engl J Med 2017;376:2415-26. 
10. Herbst RS, Baas P, Kim DW, et al. Pembrolizumab versus docetaxel for previously treated, PD-L1-positive, advanced non-small-cell lung cancer (KEYNOTE-010): a randomised controlled trial. Lancet 2016;387:1540-50.

11. Melosky B, Chu Q, Juergens R, et al. Pointed Progress in Second-Line Advanced Non-Small-Cell Lung Cancer: The Rapidly Evolving Field of Checkpoint Inhibition. J Clin Oncol 2016;34:1676-88.

12. Reck M, Rodriguez-Abreu D, Robinson AG, et al. Pembrolizumab versus Chemotherapy for PD-L1Positive Non-Small-Cell Lung Cancer. N Engl J Med 2016;375:1823-33.

13. Langer CJ, Gadgeel SM, Borghaei H, et al. Carboplatin and pemetrexed with or without pembrolizumab for advanced, non-squamous non-small-cell lung cancer: a randomised, phase 2 cohort of the open-label KEYNOTE-021 study. Lancet Oncol 2016;17:1497-508.

14. Gandhi L, Rodriguez-Abreu D, Gadgeel S, et al. Pembrolizumab plus Chemotherapy in Metastatic Non-Small-Cell Lung Cancer. N Engl J Med 2018;378:2078-92.

15. Ventola CL. Cancer Immunotherapy, Part 3: Challenges and Future Trends. P T 2017;42:514-21.

16. Rizvi NA, Hellmann MD, Snyder Aet, et al. Cancer immunology. Mutational landscape determines sensitivity to PD-1 blockade in non-small cell lung cancer. Science 2015;348:124-8.

17. Hellmann MD, Ciuleanu TE, Pluzanski, et al. Nivolumab plus Ipilimumab in Lung Cancer with a High Tumor Mutational Burden. N Engl J Med 2018;378:2093-104.

18. Taguchi F, Solomon B, Gregorc V, et al. Mass spectrometry to classify non-small-cell lung cancer patients for clinical outcome after treatment with epidermal growth factor receptor tyrosine kinase inhibitors: a multicohort crossinstitutional study. J Natl Cancer Inst 2007;99:838-46.

19. Carbone DP, Ding K, Roder H, et al. Prognostic and predictive role of the VeriStrat plasma test in patients with advanced non-small-cell lung cancer treated with erlotinib or placebo in the NCIC Clinical Trials Group BR.21 trial. J Thorac Oncol 2012;7:1653-60.

20. Chung CH, Seeley EH, Roder H, et al. Detection of tumor epidermal growth factor receptor pathway dependence by serum mass spectrometry in cancer patients. Cancer Epidemiol Biomarkers Prev 2010;19:358-65.

21. Gadgeel S, Goss G, Soria JC, et al. Evaluation of the VeriStrat $((\mathrm{R}))$ serum protein test in patients with advanced squamous cell carcinoma of the lung treated with second- line afatinib or erlotinib in the phase III LUX-Lung 8 study. Lung Cancer 2017;109:101-8.

22. Gautschi O, Dingemans AM, Crowe S, et al. VeriStrat(R) has a prognostic value for patients with advanced non-small cell lung cancer treated with erlotinib and bevacizumab in the first line: pooled analysis of SAKK19/05 and NTR528. Lung Cancer 2013;79:59-64.

23. Gregorc V, Novello S, Lazzari C, et al. Predictive value of a proteomic signature in patients with non-small-cell lung cancer treated with second-line erlotinib or chemotherapy (PROSE): a biomarker-stratified, randomised phase 3 trial. Lancet Oncol 2014;15:713-21.

24. Grossi F, Genova C, Rijavec E, et al. Prognostic role of the VeriStrat test in first line patients with non-small cell lung cancer treated with platinum-based chemotherapy. Lung Cancer 2018;117:64-9.

25. Grossi F, Rijavec E, Genova C, et al. Serum proteomic test in advanced non-squamous non-small cell lung cancer treated in first line with standard chemotherapy. Br J Cancer 2017;116:36-43.

26. Kuiper JL, Lind JS, Groen HJ, et al. VeriStrat((R)) has prognostic value in advanced stage NSCLC patients treated with erlotinib and sorafenib. Br J Cancer 2012;107:1820-5.

27. Akerley WL, Arnaud AM, Reddy B, et al. Impact of a multivariate serum-based proteomic test on physician treatment recommendations for advanced non-small-cell lung cancer. Curr Med Res Opin 2017;33:1091-7.

28. Akerley WL, Nelson RE, Cowie RH, et al. The impact of a serum based proteomic mass spectrometry test on treatment recommendations in advanced non-small-cell lung cancer. Curr Med Res Opin 2013;29:517-25.

29. Hornberger J, Hirsch FR, Li Q, et al. Outcome and economic implications of proteomic test-guided secondor third-line treatment for advanced non-small cell lung cancer: extended analysis of the PROSE trial. Lung Cancer 2015;88:223-30.

30. Page RD, Argento AC, Nash DB, et al. The Role of Proteomic Testing in Improving Prognosis And Care Planning Quality Measures for Lung Cancer. Managed Care 2017;26:37-47.

31. Milan E, Lazzari C, Anand S, et al. SAA1 is over-expressed in plasma of non small cell lung cancer patients with poor outcome after treatment with epidermal growth factor receptor tyrosine-kinase inhibitors. J Proteomics 2012;76 Spec No.:91-101.

32. Fidler MJ, Fhied C, Roder J, et al. The serum-based VeriStrat test is associated with proinflammatory reactants 
and clinical outcome in non-small cell lung cancer patients. BMC Cancer 2018;18:310.

33. Hanahan D, Weinberg RA. Hallmarkss of cancer: the next generation. Cell 2011;144:646-74.

34. FDA-NIH Biomarker Working Group. Understanding Prognostic versus Predictive Biomarkers. Available online: https://www.ncbi.nlm.nih.gov/books/NBK402284/. Accessed April 23, 2018.

35. Grossi F, Rijavec E, Biello F, et al. Evaluation of Pretreatment Serum Tests for Nivolumab Benefit in Patients with Non-Small Cell Lung Cancer. J Thorac Oncol 2017;12:S1322.

Cite this article as: Chae YK, Kim WB, Davis AA, Park LC, Anker JF, Simon NI, Rhee K, Song J, Cho A, Chang S, Ko T, Oh M, Bhave M, Viveiros P. Mass spectrometrybased serum proteomic signature as a potential biomarker for survival in patients with non-small cell lung cancer receiving immunotherapy. Transl Lung Cancer Res 2020;9(4):1015-1028. doi: 10.21037/tlcr-20-148
36. Lazzari C, Spreafico A, Bachi A, et al. Changes in plasma mass-spectral profile in course of treatment of non-small cell lung cancer patients with epidermal growth factor receptor tyrosine kinase inhibitors. J Thorac Oncol 2012;7:40-8.

37. Weber J, Martinez A, Roder H, et al. Pre-treatment patient selection for nivolumab benefit based on serum mass spectra. J Immunother Cancer 2015;3:P103.

38. Weber JS, Sznol M, Sullivan RJ, et al. A Serum Protein Signature Associated with Outcome after Anti-PD-1 Therapy in Metastatic Melanoma. Cancer Immunol Res 2018;6:79-86. 


\section{Supplementary}

Table S1 Time of the VeriStrat test relative to the treatment with ICI

\begin{tabular}{lccc}
\hline Variable & Overall $(n=32)$ & VeriStrat-classified population & VS-P $(n=14)$ \\
\cline { 3 - 4 } & & VS-G $(n=18)$ & $10(71.4)$ \\
Time of the VeriStrat test & $20(62.5)$ & $10(55.6)$ & $4(28.6)$ \\
After ICl & $12(37.5)$ & $8(44.4)$ & \\
Before ICl & & \\
\hline
\end{tabular}

Data given as No. (\%) unless otherwise indicated. ICl, immune-checkpoint inhibitor; VS-G, VeriStrat Good; VS-P, VeriStrat Poor.

Table S2 Cox proportional hazard models of progression-free survival by the overall NSCLC patients

\begin{tabular}{|c|c|c|c|c|}
\hline Variable & \multicolumn{2}{|l|}{ Univariate } & \multicolumn{2}{|c|}{ Multivariate } \\
\hline \multicolumn{5}{|l|}{ VeriStrat } \\
\hline$P$ & 1 & & 1 & \\
\hline $\mathrm{G}$ & $0.43(0.21-0.87)$ & 0.018 & $0.38(0.16-0.88)$ & 0.024 \\
\hline \multicolumn{5}{|l|}{ Gender } \\
\hline Male & 1 & & 1 & \\
\hline Female & $1.30(0.65-2.59)$ & 0.462 & $1.13(0.49-2.60)$ & 0.779 \\
\hline \multicolumn{5}{|l|}{ Age } \\
\hline $30-60$ & 1 & & 1 & \\
\hline Never & 1 & & 1 & \\
\hline Ever & $0.81(0.33-1.95)$ & 0.632 & $0.89(0.29-2.77)$ & 0.846 \\
\hline \multicolumn{5}{|l|}{ ECOG PS } \\
\hline$\leq 2$ & 1 & & 1 & \\
\hline 3,4 & $1.41(0.54-3.66)$ & 0.484 & $1.26(0.40-3.92)$ & 0.693 \\
\hline \multicolumn{5}{|l|}{ Histology } \\
\hline Squamous & 1 & & 1 & \\
\hline Non-squamous & $1.16(0.59-2.28)$ & 0.663 & $1.55(0.70-3.43)$ & 0.284 \\
\hline
\end{tabular}

a , adjusted for other six covariates. Cl, confidence interval; HR, hazard ratio; VS-G, VeriStrat Good; VS-P, VeriStrat Poor; ECOG PS, Eastern Cooperative Oncology Group Performance Status. 
Table S3 Cox proportional hazard models of overall survival by the overall NSCLC patients

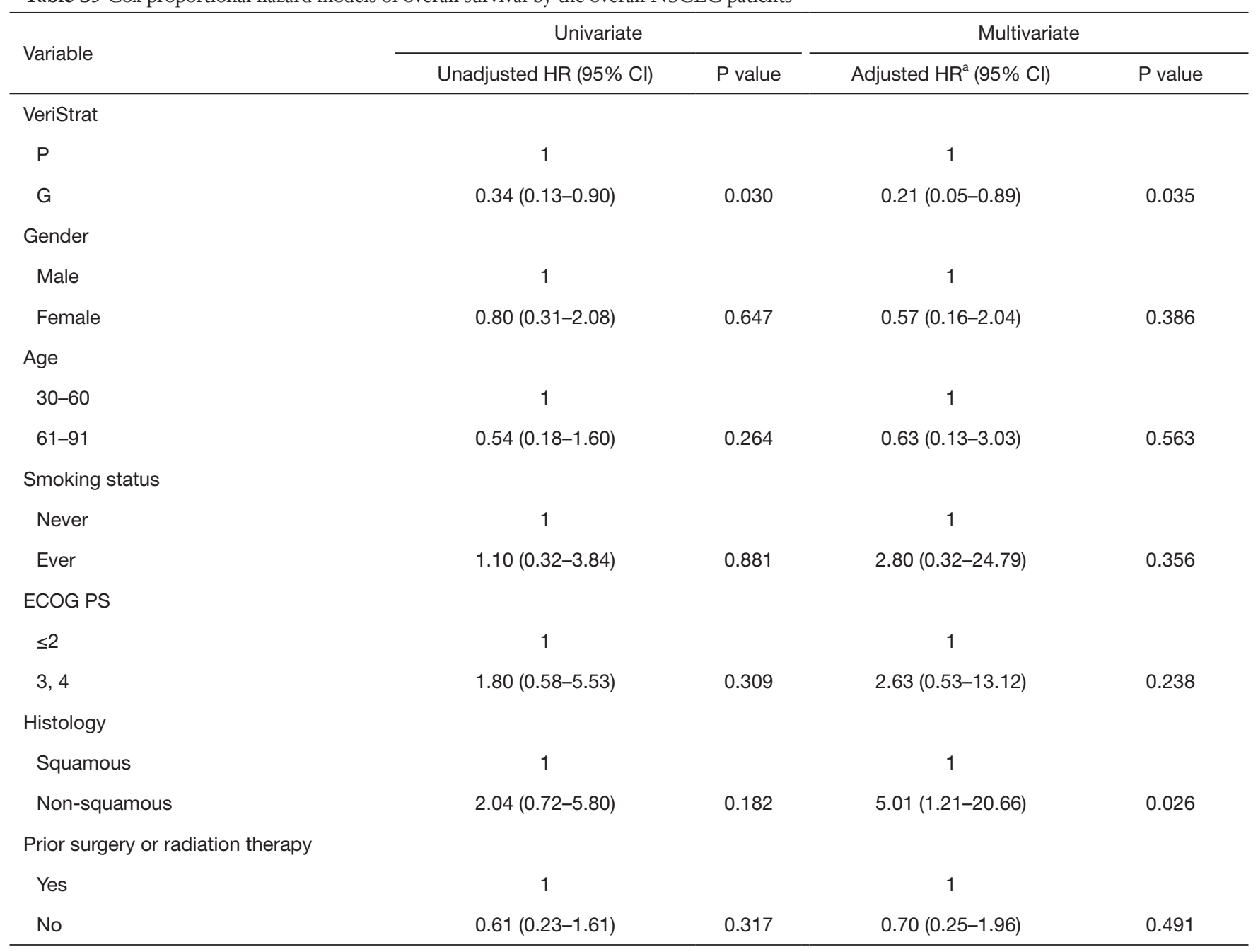

\footnotetext{
a, adjusted for other six covariates. CI, confidence interval; HR, hazard ratio; VS-G, VeriStrat Good; VS-P, VeriStrat Poor; ECOG PS, Eastern Cooperative Oncology Group Performance Status.
} 
Table S4 Cox proportional hazard models of progression-free survival by NSCLC patients treated with chemotherapy

\begin{tabular}{|c|c|c|c|c|}
\hline Variable & \multicolumn{2}{|l|}{ Univariate } & \multicolumn{2}{|c|}{ Multivariate } \\
\hline \multicolumn{5}{|l|}{ VeriStrat } \\
\hline$P$ & 1 & & 1 & \\
\hline $\mathrm{G}$ & $0.46(0.21-1.01)$ & 0.053 & $0.38(0.12-1.15)$ & 0.087 \\
\hline \multicolumn{5}{|l|}{ Gender } \\
\hline Male & 1 & & 1 & \\
\hline Female & $1.10(0.51-2.38)$ & 0.800 & $0.83(0.29-2.41)$ & 0.729 \\
\hline \multicolumn{5}{|l|}{ Age } \\
\hline $30-60$ & 1 & & 1 & \\
\hline Never & 1 & & 1 & \\
\hline Ever & 0.99 (0.38-2.59) & 0.982 & $1.00(0.31-3.27)$ & 0.997 \\
\hline \multicolumn{5}{|l|}{ ECOG PS } \\
\hline$\leq 2$ & 1 & & 1 & \\
\hline 3,4 & $1.41(0.49-4.09)$ & 0.523 & $1.62(0.37-7.08)$ & 0.519 \\
\hline \multicolumn{5}{|l|}{ Histology } \\
\hline Squamous & 1 & & 1 & \\
\hline Non-squamous & $1.68(0.80-3.54)$ & 0.174 & $2.19(0.91-5.30)$ & 0.081 \\
\hline
\end{tabular}

\footnotetext{
a, adjusted for other six covariates. CI, confidence interval; HR, hazard ratio; VS-G, VeriStrat Good; VS-P, VeriStrat Poor; ECOG PS, Eastern Cooperative Oncology Group Performance Status.
} 
Table S5 Cox proportional hazard models of overall survival by NSCLC patients treated with chemotherapy

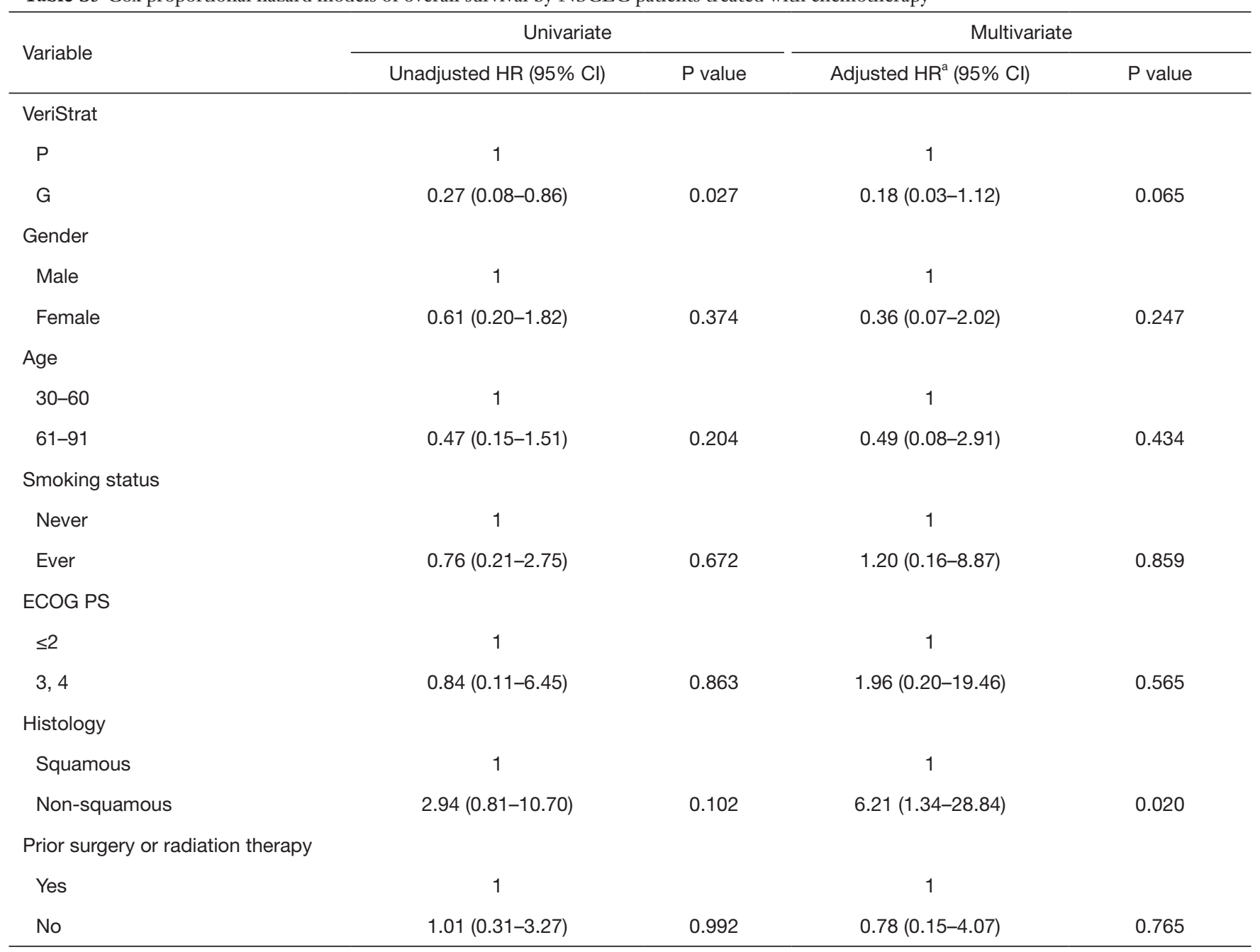

\footnotetext{
a, adjusted for other six covariates. CI, confidence interval; HR, hazard ratio; VS-G, VeriStrat Good; VS-P, VeriStrat Poor; ECOG PS, Eastern Cooperative Oncology Group Performance Status.
} 